\title{
Los archivos personales conservados en bibliotecas universitarias argentinas y su importancia para la investigación y la cultura
}

\author{
Mónica Gabriela Pené* \\ Florencia Bossié** \\ Victoria Calvente* \\ Flavia Giménez ${ }^{\star *}$
}

\begin{abstract}
Artículo recibido:
7 de mayo de 2020

Artículo aceptado:

1 de septiembre de 2020

Artículo de investigación
\end{abstract}

\section{Resumen}

Hoy día es notorio el interés que existe en la comunidad académica por los archivos personales. En el relevamiento de la literatura publicada en América Latina predominan textos sobre un fondo personal específico - a partir del cual se trabaja la noción de archivo personal, su organización, descripción y preservación- pero es escaso el relato de experiencias en bibliotecas. Esta investigación exploratoria-descriptiva tiene por objetivo conocer, mediante una encuesta, la cohabitación de fondos personales en bibliotecas universitarias argentinas de gestión pública y privada, así como la opinión de sus responsables en relación a la disponibilidad de

* Facultad de Humanidades y Ciencias de la Educación, Universidad Nacional de La Plata, Argentina mpene@fahce.unlp.edu.arvvcalvente@fahce.unlp.edu.ar

** Biblioteca Pública, Universidad Nacional de La Plata, Argentina

florencia.bossie@biblio.unlp.edu.ar flavia.gimenez@biblio.unlp.edu.ar

INVESTIGACIÓN BIBLIOTECOLÓGICA, vol. 35, núm. 86, enero/marzo, 2021, México, ISSN: 2448-8321 pp. 143-160 
este tipo de documentos en sus instituciones. La población de estudio estuvo conformada por las bibliotecas pertenecientes a 131 instituciones de educación superior. Para la recolección de datos se elaboró un cuestionario. Sobre 35 respuestas obtenidas, los resultados muestran que la mitad de las bibliotecas poseen este tipo de fondo, la mayoría ha confeccionado algún instrumento de descripción, $40 \%$ ha iniciado su digitalización, 17 \% cuenta con políticas en materia archivística, y $80 \%$ de los responsables de biblioteca valoran positivamente el ingreso de archivos personales; todas señales de que las bibliotecas están replanteando sus prácticas y políticas frente a estas valiosas fuentes de información.

Palabras clave: Archivos Personales; Colecciones Especiales; Bibliotecas Universitarias; Argentina

Personal archives preserved in Argentine university libraries and their importance for research and culture Mónica Gabriela Pené, Florencia Bossié, Victoria Calvente, and Flavia Giménez

\section{Abstract}

Nowadays, it is notorious the interest that exists in the academic community for personal archives. In the survey of literature published in Latin America, texts on a specific personal archive predominate -from which the notion of personal archive is elaborated, as well as its organization, description and preservation-, but experiences in libraries are scarce. The objective of this exploratory-descriptive research is to to know, through a survey, the cohabitation of personal archives in Argentine public and private university libraries, as well as the opinion of their authorities regarding the availability of this type of documents in their institutions. The population of study was made up of libraries belonging to 131 institutions of higher education, and for data collection a questionnaire was developed. With 35 responses obtained, the results show that half of the libraries have this type of material, that most of them have made an instrument of description, that $40 \%$ have started their digitization and $17 \%$ have archival policies, and that $80 \%$ of library managers value 
positively the reception of personal papers. Signs all of them, that libraries are rethinking their practices and policies against these valuable sources of information.

Keywords: Personal Archives; Special Collections; University Libraries; Argentina

\section{INTRODUCCIÓN}

A 1 definir los archivos personales, Portela Filgueiras (2017), Rosell León (2006) y Svicero (2013) coinciden en señalar que la bibliografía sobre estos fondos es escasa, no han sido analizados profundamente y es poca la información que figura sobre ellos en los manuales clásicos de la archivología, lo que nos lleva a pensar que constituyen un campo poco conocido. Englobado en la esfera de lo privado, este conjunto de documentos generados y/o reunidos por una persona a lo largo de su vida para sus propias necesidades, en el marco de sus actividades personales y profesionales, es testimonio fehaciente de sus motivaciones, inquietudes e intereses (Bergaglio y Pené, 2003). Amplía la mirada no sólo sobre su creador o su obra, sino también de la época en que le tocó vivir, convirtiéndose en una valiosa fuente de información para la investigación, cuyo contenido puede cubrir los vacíos documentales que existen en otros archivos (Portela Filgueiras, 2017), por lo que su reunión y preservación debería ser tema de interés público.

Por su parte, Mastropierro Tellechea (2006: 17) define al archivo personal como una "colección de documentos referidos a la actividad de una persona, preparada profesionalmente como testimonio para su memoria". Consideramos que colección, en el caso de los archivos personales, no debería ser el primer término a utilizar para su conceptualización, sino más bien conjunto de documentos. La colección implica una reunión artificial de documentos de cualquier procedencia, agrupados por su creador en función de una característica común (tipo de documento, soportes, idioma, temática, etc.), creados como fruto del saber y destinados a su goce y/o difusión. Se diferencia del concepto de fondo de archivo en que éste constituye un conjunto de documentos procedentes de la actividad de una persona cuya reunión es consecuencia de un proceso natural en el que el productor genera y conserva esos fondos de forma progresiva y constante (Cruz Mundet, 2003; Díaz Rodríguez, 2008). Esta diferencia conceptual marca el camino hacia el tratamiento que los fondos personales han de recibir, ya que, como bien señala Santoyo Bastida (2014: 41), será necesario "identificar adecuadamente la documentación gene- 
rada por las funciones o actividades del personaje, la cual sería considerada el fondo, y lo que llamaríamos colección, que serían los documentos reunidos de manera voluntaria y que provienen de diversas fuentes".

En cuanto a la preparación profesional a la que refiere Mastropierro Tellechea (2006), es importante recalcar la diferencia entre fondo personal y archivo personal, basada justamente en el trabajo profesional que se requiere para organizar, describir, preservar y brindar acceso a dichas fuentes. Si partimos de la noción de fondo como un conjunto de documentos de diverso tipo, sean publicados o inéditos, existentes en una institución, con un origen y procedencia común, y avanzamos hacia la definición de fondo de archivo como aquel conjunto documental procedente de la actividad de un organismo o un individuo que lo genera y conserva de forma natural, podemos decir que un fondo personal es aquel que custodia documentos en cualquier formato y/o soporte generados en el contexto de las actividades de una persona, y que sólo cuando este fondo personal es organizado y la recuperación de información contenida en él es posible, puede ser llamado archivo personal (Cruz Mundet, 2003; Díaz Rodríguez, 2008).

Por lo general, el creador es una personalidad de la vida pública -un escritor, artista, político, religioso o intelectual- quien reúne una heterogénea tipología documental (cartas, originales manuscritos o mecanografiados de obras escritas, fotografías, recortes de prensa, dibujos, sellos, entre otros), organizada mediante criterios que mejor satisfagan sus necesidades de relacionarse con dichos documentos (Prieto, 2016). Estas particularidades presentan un desafío para su tratamiento, al requerir técnicas de disciplinas relacionadas pero diferentes como son la archivología, la bibliotecología y la museología. Por permitir distintos abordajes, los fondos personales suelen encontrarse tanto en centros de archivo como en bibliotecas, pues su inclusión no depende tanto de la institución receptora sino más bien del deseo del donante (Rucio Zamorano, 2012). En el caso específico de las bibliotecas, suelen integrar lo que se conoce como colecciones especiales, o lo que algunos autores denominan también colecciones ocultas (RBM, 2004) por su falta de tratamiento -o erróneo tratamiento- ante el desconocimiento sobre las técnicas adecuadas para gestionar su procesamiento.

De un tiempo a esta parte, es notorio el interés que el archivo personal ha despertado en la comunidad académica y en la sociedad en general. En el mundo anglosajón y europeo, vemos que este interés se remonta a la segunda mitad del siglo pasado (Pollard, 2001; Vautier, 2015). No obstante, en América Latina recién es visible a comienzos del siglo XXI, observándose hoy día una corriente de investigación que crece paulatinamente pero que aún no ha generado un corpus consolidado de conocimiento en 
el tema (Pulgarín Gallego, 2017). Con Brasil en el primer puesto de países con investigaciones en este tópico, seguido por Argentina y México, notamos que la mayoría de las publicaciones hacen referencia a un fondo personal en particular y, a partir de él, se trabaja la noción de archivo personal, su organización, descripción, difusión y preservación. No siempre los trabajos son escritos por profesionales de las ciencias de la información, sino por especialistas de otras áreas del saber que utilizan los fondos personales para sus propias investigaciones disciplinares (Cucuzza, 2007; Goldchluk, 2010; Largo Vargas, 2019; Ramos Villalobos, 2017). Por otra parte, encontramos escasa literatura sobre la experiencia de archivos personales en bibliotecas, predominando textos relacionados con fondos disponibles en bibliotecas nacionales (Díaz Rodríguez, 2013; Guerra, 2016; Sanguinetti Epifanio, 2018; Zegers, 2006). En lo que respecta al ámbito universitario, se observan algunos trabajos sobre fondos personales localizados en archivos universitarios (Albornoz Rugeles, 2018; Guelfi Campos, 2014; Ríos, 2008), pero resultan escasos los textos que demuestren la existencia de archivos personales en bibliotecas universitarias (Giménez y Bossié, 2013; Gómez Gómez y Villanueva Bazán, 2007; Santos, 2018; Pené y Unzurrunzaga, 2015).

Ante este panorama, nos preguntamos si es común que existan fondos personales en las bibliotecas universitarias. Nuestra hipótesis es que este tipo de fondo es más frecuente de lo que pensamos en esta clase de unidades de información. Partiendo de esta idea, la presente investigación tiene por objetivo conocer, mediante la literatura publicada y la realización de una encuesta exploratoria, la cohabitación de archivos y/o fondos personales en bibliotecas universitarias de gestión pública y privada localizadas en Argentina, determinar su nivel de tratamiento y digitalización, así como saber la opinión de los responsables respecto a disponer y difundir documentos de archivo en la biblioteca universitaria. Se busca poner sobre la mesa un tema de interés actual por el que aún hay mucho por investigar.

\section{Metodología}

Para llevar adelante esta investigación exploratoria-descriptiva acerca de la existencia y disponibilidad de archivos personales en bibliotecas universitarias argentinas utilizamos como técnica la encuesta. Se tomó como población de estudio a las bibliotecas pertenecientes a universidades e institutos de educación superior tanto de gestión pública como privada, sean éstas centrales de facultad o de universidad, departamentales o de centros de investigación, 
existentes en la República Argentina. Dado que la presente investigación tuvo por limitación la falta de un listado autorizado y actualizado de todas las bibliotecas existentes en las instituciones en estudio, resultó imposible obtener el dato del total del universo. Para paliar esa situación, optamos por considerar-como mínimo- la existencia de una biblioteca por universidad.

Para determinar la cantidad de universidades existentes en Argentina tomamos como punto de partida el listado de instituciones de educación superior provisto por el Ministerio de Educación de la Nación (https://www.argentina.gob.ar/educacion/universidades/consejos-regionales-es), a partir de la clasificación de los Consejos Regionales de Planificación de la Educación Superior (CPRES), que dividen a las instituciones universitarias de gestión pública y privada en siete regiones, a saber: Metropolitano, Bonaerense, Centro, Nordeste, Noroeste, Nuevo Cuyo, y Sur. El listado inicial constaba de 160 instituciones, pero debido a que algunas de ellas aparecían representadas por sus sedes (por ejemplo, la Universidad Tecnológica Nacional -UTN-, cuyas sedes regionales se contabilizaban por separado), decidimos realizar una depuración de dicho listado, dejando una fila por cada universidad (es decir, la UTN se contabilizó una sola vez, más allá de las sedes que posee). Así, el listado final contó con 131 instituciones repartidas a lo largo del país.

La encuesta fue realizada vía internet, mediante un cuestionario en línea autoadministrado, durante el periodo comprendido entre el 1 de febrero y el 31 de julio de 2019. Para su difusión y conocimiento entre la comunidad, redactamos un mensaje que se envió en dos oportunidades -febrero y mayo de 2019- a las siguientes listas de interés asociadas a bibliotecas: ABGRA (Asociación de Bibliotecarios Graduados de la República Argentina), BIBUNAR (Bibliotecas Universitarias Argentinas), SIU-Bibliotecas (Sistema de Información Universitaria), REDIAB (Red Interuniversitaria Argentina de Bibliotecas) y UNLP-Roble (red de bibliotecas de la Universidad Nacional de La Plata).

Para la recolección de los datos diseñamos un cuestionario semiestructurado, basado en el formulario de ingreso de datos utilizado en el Portal Orbescrito (http://orbescrito.fahce.unlp.edu.ar/), un sitio dedicado a relevar archivos de escritores y sostenido por nuestro grupo de investigación de la UNLP. Generado mediante la herramienta Formularios de Google, el cuestionario contó con preguntas cerradas que posibilitaron respuestas precodificadas, así como con preguntas abiertas que permitieron a las personas encuestadas elaborar las respuestas con sus propias palabras, aportando así elementos cualitativos a la investigación. El instrumento se estructuró en cuatro secciones: la primera para consignar datos institucionales (tipo de institución, nombre de la institución, nombre de la biblioteca, tipo de biblioteca, persona responsable, web, medios de contacto), la segunda para indagar acerca de la existencia de archivos personales en la institución, la tercera para 
solicitar datos sobre los archivos personales existentes (nombre del archivo, tipos de documentos presentes, grado de digitalización, herramientas de difusión/nivel de descripción, condiciones de acceso, formación del personal, existencia de política de ingreso de material de archivo), y la última para conocer la opinión de los responsables de las bibliotecas en cuanto a la existencia de esta clase de fondos en este tipo de unidades de información. La realización de una prueba piloto permitió detectar consultas poco claras y mejorar su formulación. Para el análisis de los datos utilizamos la planilla de cálculo que devuelve el formulario generado con la herramienta de Google.

Considerando los objetivos perseguidos en la investigación, a continuación se detallan las variables definidas para el estudio y los valores que éstas pueden asumir:

- Tipo de institución encuestada (universidad de gestión pública o de gestión privada).

- Tipo de biblioteca universitaria (biblioteca central de universidad, biblioteca central de facultad, biblioteca departamental, biblioteca de centro de investigación, otro).

- Existencia de archivos personales en la institución (sí, no, se desconoce).

- Características particulares de esos archivos:

- Nombre del archivo.

- Tipos de documentos presentes (documentación personal -documento de identidad, pasaporte, licencia de conducir, etc.-, correspondencia, fotografías, recortes de prensa, manuscritos, textos mecanografiados, grabaciones sonoras, videos, otros).

- Grado de digitalización (digitalizados en su totalidad, digitalizados parcialmente, no digitalizados, se desconoce).

- Herramientas de difusión/nivel de descripción (existencia de guía del archivo, inventario en archivo de texto, inventario en planilla de cálculo, base de datos de acceso restringido, registro en el catálogo de la biblioteca, mención en el sitio web de la biblioteca, difusión en el Boletín de Noticias, realización de muestras con los fondos custodiados).

- Condiciones de acceso (texto libre).

- Formación del personal a su cuidado (archivólogo, bibliotecólogo con formación/capacitación en archivología, bibliotecólogo sin formación/capacitación en archivología, idóneo, otro).

- Existencia de política de ingreso de material de archivo (texto libre).

- Opinión sobre la existencia de archivos personales en la biblioteca universitaria (texto libre). 


\section{RESULTADOS Y DisCUSIÓN}

Se recibieron 35 respuestas, varias de ellas provenientes de bibliotecas pertenecientes a una misma universidad. Si se tienen en cuenta las 131 instituciones de educación superior identificadas en los CPRES, tan sólo 22 están comprendidas en dichas respuestas, lo que representa $17 \%$ del universo en estudio. Si bien el porcentaje de respuesta fue bajo ${ }^{1}$ y no permite obtener un grado de confiabilidad adecuado, consideramos que los resultados proporcionados en el presente trabajo contribuyen al llamado de atención que pretende visibilizar los archivos personales existentes en las bibliotecas universitarias de nuestro país.

En lo que respecta a las regiones CPRES, todas tuvieron representación aunque con distinta tasa de respuesta (Figura 1), destacándose la región del Centro donde casi la mitad de las instituciones participaron del relevamiento (42\%, 8 de 19). Si bien estos valores no permiten extrapolar los resultados al conjunto de las bibliotecas universitarias argentinas, posibilitan una valoración cualitativa de éstos, ayudando a comprender mejor el panorama de los archivos personales en las bibliotecas en estudio.

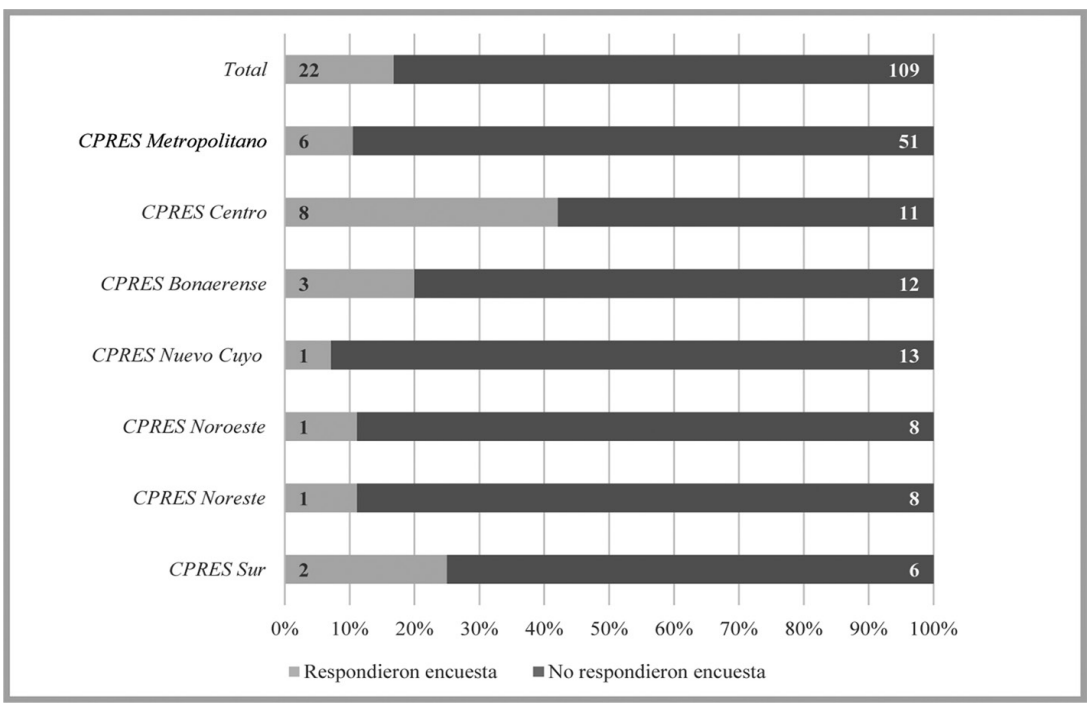

Figura 1. Tasa de respuesta a la encuesta realizada según región CPRES

Fuente: elaboración propia

1 Culminado el plazo de la encuesta, y mediante intercambios con colegas, pudimos determinar que diversas bibliotecas no contestaron la consulta porque consideraron que, al no tener fondos de archivo, no tenía utilidad su participación en la misma, hecho que contribuyó a reducir el número de respuestas recibidas. 
Ahora bien, analizando el total de respuestas, $86 \%$ (30) provino de universidades públicas y $14 \%$ (5) restante de universidades privadas. En cuanto a la tipología de las bibliotecas que respondieron la encuesta (Figura 2), predominan las centrales de universidad $(43 \%, 15)$ y de facultad $(40 \%, 14)$. Como observamos en la literatura consultada, la llegada de este tipo de materiales a las bibliotecas puede deberse a diferentes razones, entre ellas la decisión del donante -sea éste el productor de los documentos, sus familiares y herederos, o bien el investigador a quien se le confió el fondo-, lo que explicaría en parte la diversidad de destinos de los fondos personales en el contexto de las universidades argentinas.

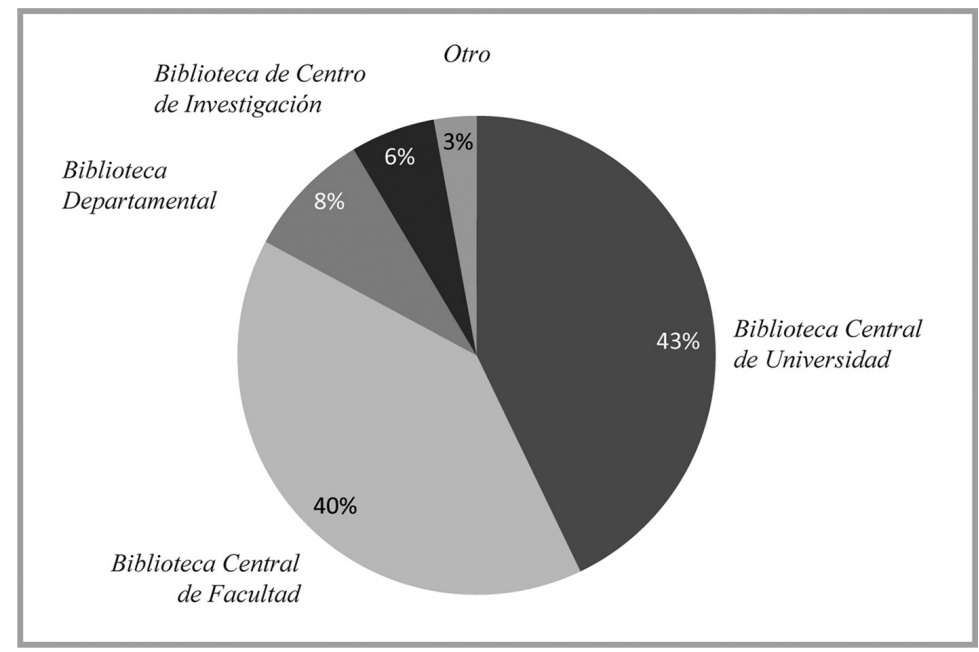

Figura 2. Tipo de biblioteca universitaria involucrada en el estudio Fuente: elaboración propia

$\mathrm{Al}$ consultar a las bibliotecas sobre la existencia de archivos personales como parte de sus colecciones, destacamos que $51 \%$ (18) haya respondido afirmativamente. Si bien el número de la muestra analizada es bajo, el hecho de que la mitad de las bibliotecas encuestadas señalara la disponibilidad de fondos personales como parte de sus acervos abre la posibilidad de que la hipótesis del trabajo sea cierta y que los fondos personales sean materiales frecuentes en las bibliotecas universitarias.

Con base en las respuestas obtenidas, estas 18 bibliotecas reúnen un total de 58 fondos donde predominan aquellos relacionados con personajes destacados del mundo de la política, el periodismo y la historia local y nacional $(48 \%, 28)$-como es el caso de Arturo Costa Álvarez, Federico Pinedo, Gino Germani, Gregorio de Laferrere, José María Aricó o Santiago Senén 
González-. Mientras que 24 \% (14) de los fondos identificados refieren a personas relevantes vinculadas al mundo de la educación y particularmente a la vida universitaria -como los de Gregorio Weinberg, Joaquín V. González, Juan Carlos Tedesco, Miguel Cangiano, Rodolfo Kusch, Víctor Mercante y Virginia Erhart-, 21 \% (12) corresponde a figuras relacionadas a las artes, la cultura, la literatura y la música -como los de Almafuerte (Pedro B. Palacios), Edgardo Antonio Vigo, Jaime Rest, Manuel Puig y Néstor Ortíz Oderigo-, y $7 \%$ (4) a personas destacadas de la comunidad local.

Los archivos de las 18 bibliotecas suelen reunir una heterogénea tipología de documentos (Figura 3), entre los que predominan la correspondencia (78 \%, 14), los manuscritos $(67 \%, 12)$, los textos mecanografiados $(67 \%$, $12)$, los recortes de prensa $(67 \%, 12)$ y las fotografías $(61 \%, 11)$. Esto condice con lo señalado en la bibliografía consultada, siendo una característica típica de los archivos personales la presencia de una diversidad de tipos y formatos documentales, lo que aporta valor pero también complejidad al tratamiento de estos fondos.

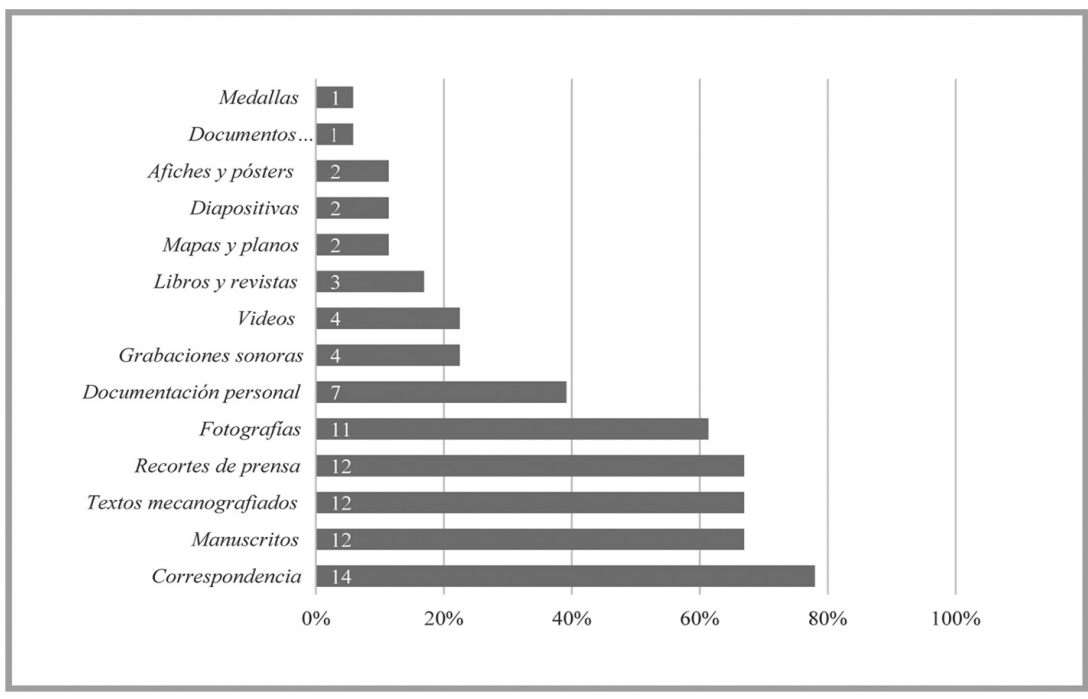

Figura 3. Tipos documentales disponibles en los fondos de archivo existentes en las bibliotecas universitarias relevadas Fuente: elaboración propia

Observamos, asimismo, que más de la mitad de las bibliotecas que poseen fondos personales aún no ha iniciado su digitalización $(61 \%, 11)$, ya sea porque se encuentran en la etapa de confección y acondicionamiento de los instrumentos de descripción o porque no disponen de personal y equipamiento 
necesario para llevar adelante la tarea. No obstante, $6 \%$ (1) de los fondos digitalizados totalmente y $33 \%$ (6) de los digitalizados parcialmente muestran un interés creciente -aunque paulatino- en la digitalización de los fondos, ya sea con fines de difusión, acceso y/o preservación.

En cuanto a las condiciones de acceso, por lo general son materiales disponibles para consulta en sala por parte de docentes e investigadores, bajo presentación de identificación personal y cita previa. Aunque existen fondos digitalizados, la consulta en línea a través de internet de los documentos a texto completo aún no es posible en la mayoría de los casos.

Con la intención de dar a conocer a los usuarios el contenido de los fondos personales disponibles, las bibliotecas han elaborado diversos instrumentos de descripción. Resalta la preeminencia del inventario en planilla de cálculo como el instrumento más usual $(67 \%, 12)$-generalmente de consulta en la sede de la biblioteca-, seguido del registro en el catálogo en línea de la biblioteca $(28 \%, 5)$. La guía de archivo ha sido elegida por el $17 \%$ (3) de las bibliotecas relevadas, al igual que el inventario en archivo de texto; mientras que la base de datos de acceso restringido sólo se observa en $11 \%$ (2) de los casos analizados. Cabe señalar que la mención del archivo en el sitio web de la biblioteca es una constante que observamos como medio para la difusión de los fondos personales $(50 \%, 9)$, junto con la exhibición de los materiales en distintas muestras $(17 \%, 3)$ y su inclusión en los boletines de noticias $(17 \%, 3)$. Estos trabajos relacionados al procesamiento de los documentos de archivo son señales claras de que las bibliotecas comienzan a replantearse sus prácticas tradicionales, ampliando sus descripciones al incorporar técnicas propias de la archivología.

De las 18 bibliotecas universitarias que poseen archivos personales, sólo una no contestó la pregunta acerca de la formación del personal que trabaja con estos fondos. En cuanto a las demás, observamos que 17 \% (3) cuenta con un archivólogo entre su plantel, mientras que 44 \% (8) manifiesta contar con bibliotecólogos que poseen formación/capacitación en archivología. Considerando los porcentajes antes señalados, podemos decir que poco más de la mitad de las instituciones poseen recursos humanos capacitados para llevar adelante las tareas de organización y descripción de fondos de archivo, hecho que condice con los porcentajes de bibliotecas que han elaborado instrumentos de descripción archivística. No obstante, en el resto de las bibliotecas relevadas sólo existen bibliotecólogos sin formación (22 \%, 4), o bien personal idóneo $(11 \%, 2)$. Si bien los porcentajes observados resultan alentadores, también dejan de manifiesto la necesidad de continuar con capacitaciones en temáticas archivísticas en el contexto de las bibliotecas universitarias, así como la incorporación específica de archivólogos. Por otro 
lado, entendiendo que los archivos personales reúnen materiales heterogéneos, pocas son las instituciones que cuentan con un equipo multidisciplinar para hacer frente a su procesamiento $(6 \%, 1)$, destacándose el caso de una biblioteca que posee un archivólogo, un bibliotecólogo con formación en archivología y un historiador.

En lo que respecta a la existencia de políticas bibliotecarias que contemplen el ingreso de fondos archivísticos en las bibliotecas universitarias, podemos decir que todas las instituciones que declararon no poseer este tipo de fondos concuerdan en no mencionar a los mismos como parte de las colecciones comprendidas en sus políticas. Por su parte, de las unidades de información relevadas que sí poseen archivos personales, $83 \%$ (15) no cuenta con políticas explícitas al respecto y tan sólo 17 \% (3) ha elaborado textos en este sentido, que por lo general indican el alcance de los fondos que interesa reunir y las formas establecidas para el ingreso de materiales. No es de extrañar el escaso porcentaje de unidades de información que han elaborado este tipo de documento, sobre todo si se considera que es bastante común que las bibliotecas no dediquen tiempo a la redacción de políticas relacionadas a la gestión y desarrollo de sus colecciones (Aguado de Costa, 2011).

Por último, solicitamos a los responsables de las bibliotecas encuestadas (35), tuvieran o no fondos archivísticos, que brindaran su opinión acerca de la inclusión de este tipo de documentos en sus instituciones. El 80 \% (28) valoró como positivo el ingreso de archivos personales, más aún cuando los mismos están relacionados a temáticas de interés de la facultad y/o universidad, o son fondos generados/reunidos por docentes e investigadores de la casa de estudios. Las razones principales para dar espacio a este tipo de materiales en las bibliotecas universitarias parecieran tener que ver con la necesidad de contar con fuentes de interés para la investigación $(51 \%, 18)$ el resguardo de la memoria institucional y académica $(20 \%, 7)$ y la consideración de que estos fondos representan parte del patrimonio documental $(9 \%, 3)$. Por las razones antes expuestas, los responsables consideran pertinente su reunión, registro, difusión y preservación, sobre todo cuando en las propias universidades no existan centros de archivos constituidos que pudieran recibir estos valiosos documentos.

\section{Conclusiones}

Como ha podido observarse a lo largo del presente trabajo, el creciente interés por los archivos personales ha llevado a las bibliotecas argentinas a replantearse sus prácticas y políticas de gestión frente a este tipo de documentos. 
Adormecidos durante mucho tiempo en algún rincón de estas instituciones, los archivos personales comienzan a ser visibles. Esto es notorio desde la reciente literatura publicada, así como por la frecuencia de eventos en los que se discute el tema en nuestro país, síntomas de un proceso que se ha iniciado para otorgarle a este tipo de archivo un lugar en el denominado patrimonio documental, asignándole valor como fuente de información relevante para la investigación.

Ante este panorama, las bibliotecas universitarias no han permanecido ajenas. Los resultados de la encuesta realizada ponen de manifiesto que algo está cambiando en ellas. Si bien no ha podido corroborarse la hipótesis del trabajo por la baja tasa de respuesta, el hecho de que la mitad de las unidades de información relevadas confirmen la existencia de este tipo de fondo como parte de su acervo sirve como una llamada de atención para pensar y reflexionar sobre los archivos personales en el contexto de las bibliotecas universitarias argentinas. Aún queda mucho camino por recorrer. La mayoría de estas instituciones ha confeccionado algún instrumento de descripción y varias han iniciado procesos de digitalización, pero son escasas las que cuentan con políticas establecidas en materia de fondos de archivo, además de que no todos los instrumentos construidos están accesibles desde sus sitios web. No obstante, la valoración positiva por parte de los responsables de biblioteca acerca del ingreso de archivos personales, sea porque los consideran útiles para la investigación, parte de la memoria institucional y académica o bien del patrimonio documental del país, y sin duda redundará en un beneficio para estos papeles que han esperado demasiado tiempo para salir a la luz.

Sabemos que pensar el archivo en el contexto de la biblioteca no es tarea fácil, ya que la formación académica de los bibliotecólogos no es suficiente para abordar este tipo de documentos, a lo que se suma generalmente la falta de personal -sobre todo especializado y multidisciplinar- en la mayoría de las instituciones, tal como quedó expuesto en los casos analizados. Si además tenemos en cuenta que junto con los fondos bibliográficos y documentales pueden coexistir objetos de museo, la situación se complejiza aún más. Se vuelve necesario que la bibliotecología, la archivología y la museología actúen en conjunto a fin de garantizar un tratamiento integral a los materiales (Yarrow, Clubb y Draper, 2009).

Una situación ideal para nuestras instituciones sería contar con un equipo de trabajo interdisciplinario formado por archivólogos, bibliotecólogos y museólogos, que con sus saberes relacionados favorezcan un mejor tratamiento de los documentos que luego son puestos a disposición del público. Pero las realidades institucionales pocas veces se acercan a la situación ideal, por lo que consideramos indispensable la capacitación específica y, sobre todo, el 
trabajo en colaboración entre las diferentes profesiones y los diversos tipos de instituciones culturales. Sólo así las prácticas que se llevan adelante en las bibliotecas, en materia de archivos personales, incorporarán la perspectiva archivística para otorgarles la amplitud necesaria para visibilizar y a la vez dar valor a los documentos de archivo albergados en ellas.

Para finalizar, queremos resaltar la necesidad de que bibliotecólogos y archivólogos dediquen mayor atención a este tipo particular de archivo, compartiendo experiencias y saberes a través de la publicación de trabajos en el contexto de las ciencias de la información, que aporten a la conformación de un corpus disciplinar que favorezca un estudio más profundo y acabado de estas valiosas fuentes de información que constituyen los archivos personales.

Agradecimientos

Agradecemos a las siguientes instituciones por participar en la encuesta y hacer posible la presente investigación: Instituto Tecnológico de Buenos Aires. Biblioteca "Dr. Amadeo J. Galli”; Universidad Adventista del Plata. Biblioteca "E. Irving Mohr”; Universidad Católica de Córdoba. Sistema de Bibliotecas; Universidad de Buenos Aires. Facultad de Agronomía. Biblioteca "Arata"; Universidad de San Andrés. Biblioteca "Max von Buch"; Universidad Nacional Arturo Jauretche. Biblioteca Central; Universidad Nacional de Córdoba. Biblioteca "José María Aricó”; Universidad Nacional de Córdoba. Facultad de Filosofía y Humanidades. Biblioteca "Elma Kohlmeyer de Estrabou”; Universidad Nacional de Córdoba. Observatorio Astronómico de Córdoba. Biblioteca "Roberto F. Sisteró"; Universidad Nacional de Entre Ríos. Facultad de Ingeniería. Biblioteca "Dr. Eduardo A. Barbagelata”; Universidad Nacional de La Pampa. Facultad de Ciencias Veterinarias. Biblioteca Informática; Universidad Nacional de La Plata. Biblioteca Pública; Universidad Nacional de La Plata. Facultad de Ciencias Económicas. Biblioteca "Raúl A. Granoni"; Universidad Nacional de La Plata. Facultad de Ciencias Naturales y Museo. Biblioteca "Florentino Ameghino"; Universidad Nacional de La Plata. Facultad de Ciencias Agrarias y Forestales, y Facultad de Ciencias Veterinarias. Biblioteca Conjunta; Universidad Nacional de La Plata. Facultad de Humanidades y Ciencias de la Educación. Biblioteca "Profesor Guillermo Obiols"; Universidad Nacional de los Comechingones. Biblioteca; Universidad Nacional de Rafaela. Biblioteca; Universidad Nacional de Río Negro. Sede Alto Valle y Valle Medio. Biblioteca central; Universidad Nacional de Rosario. Facultad de Humanidades y Artes. Escuela de Letras. Biblioteca de Letras; Universidad Nacional de Rosario. Facultad de Psicología. Biblioteca "Dr. Juan Carlos Gardella”; Universidad Nacional de Rosario. 
Instituto Politécnico Superior General San Martín. Biblioteca "Domingo Faustino Sarmiento"; Universidad Nacional de Tres de Febrero. Biblioteca y Centro de Documentación; Universidad Nacional de Tucumán. Facultad de Medicina. Biblioteca "Prof. Dr. Alfredo Amenábar"; Universidad Nacional de Villa María. Biblioteca Central "Vicerrector Ricardo Podestá"; Universidad Nacional del Litoral. Centro Universitario "Pablo Vrillaud", Sede Reconquista. Biblioteca; Universidad Nacional del Litoral. Centro Universitario Gálvez. Biblioteca; Universidad Nacional del Litoral. Consorcio de las facultades de Bioquímica y Ciencias Biológicas, e Ingeniería y Ciencias

Hídricas y Ciencias Médicas. Biblioteca centralizada "Ezio Emiliani”; Universidad Nacional del Litoral. Facultad de Ciencias Económicas. Biblioteca; Universidad Nacional del Nordeste. Biblioteca del Instituto de Investigaciones Geohistóricas; Universidad Nacional del Nordeste. Dirección General de Bibliotecas. Biblioteca Central; Universidad Nacional del Nordeste. Facultad de Ciencias Veterinarias. Biblioteca Agropecuaria; Universidad Nacional del Sur. Departamento de Humanidades. Biblioteca "Arturo Marasso"; Universidad Pedagógica Nacional. Biblioteca Central UNIPE; Universidad Torcuato

Di Tella. Biblioteca.

\section{REFERENCIAS}

Aguado de Costa, Amelia. 2011. Gestión de colecciones. Buenos Aires: Alfagrama.

Albornoz Rugeles, Cristina. 2018. “¡Eran seis mil documentos! El archivo documental que Rogelio Salmona constituyó para la Universidad de los Andes”. DEARQ: Revista de Arquitectura de la Universidad de los Andes (22): 170-175. https://doi.org/10.18389/dearq22.2018.09

Bergaglio, Carolina y Mónica G. Pené. 2003. "Memoria colectiva: Su generación a partir de colecciones privadas", en Actas del V Congreso de Archivología del Mercosur, "Política archivística e identidad". Huerta Grande : Federación de Archiveros de la República Argentina; Asociación de Archiveros de la Provincia de Córdoba.

Cruz Mundet, José Ramón. 2003. Manual de archivística. 5a. ed. Madrid: Fundación Germán Sánchez Ruipérez.

Cucuzza, Héctor Rubén. 2007. "Allons enfants de la patrie: el archivo personal de Anunciada Mastelli”. Archivos de Ciencias de la Educación (1): 109-130. https:/www.archivosdeciencias.fahce.unlp.edu.ar/article/view/ARCHv01n01a05

Díaz Rodríguez, María del Rosario. 2008. "Entre bibliotecas y archivos: Los transgresores archivos personales". Biblioteca: Anales de investigación 4. https://dialnet.unirioja.es/servlet/articulo? codigo=6099056

Díaz Rodríguez, María del Rosario. 2013. "Archivos personales en Cuba: El fondo Fernando Ortiz". Biblioteca: Anales de investigación 8-9 (8-9): 20-30. https://dialnet.unirioja.es/servlet/articulo? codigo $=5704457$ 
Giménez, Flavia y Florencia Bossié. 2013. “Archivos personales y manuscritos en la Biblioteca Pública de la Universidad Nacional de La Plata: Propuestas para su organización y su descripción”, en Actas de las VI Jornadas Internacionales de Filología y Lingüistica y Primeras de Crítica Genética "Las lenguas del archivo". La Plata: FAHCE.

http://jornadasfilologiaylinguistica.fahce.unlp.edu.ar

Goldchluk, Graciela. 2010. “¿Dónde sucede la literatura? Libro, manuscrito y archivo en Manuel Puig y Mario Bellatin”. El bilo de la fábula 7 (8-9): 93-100. https://doi.org/10.14409/hf.v1i8/9.1919

Gómez Gómez, Alma Leticia y Gustavo Villanueva Bazán. 2007. "Ezequiel A. Chávez: Un archivo automatizado". Biblioteca Universitaria: Revista de la Dirección de Bibliotecas de la UNAM 10 (2): 169-180. http://www.revistas.unam.mx/index.php/rbu/article/view/24714/23193

Guelfi Campos, José Francisco. 2014. "Preservando a memória da ciência brasileira: os arquivos pessoais de professores e pesquisadores da Universidade de São Paulo”. Tesis de maestría, Universidade de Sao Paulo, Faculdade de Filosofía, Letras e Ciencias Humanas. https://doi.org/10.11606/D.8.2014.tde-11122014-190123

Guerra, Ana. 2016. "A 10 años de trabajo con archivos y colecciones particulares en la Biblioteca Nacional (Argentina)”. Revista Electrónica de Fuentes y Archivos 7 (7): 302-308. https://refa.org.ar/file.php?tipo=Contenido \&id $=163$

Largo Vargas, Joan Manuel. 2019. "Ignacio Torres Giraldo y los lenguajes políticos en Colombia, 1893-1968”. Historia y Memoria (18): 177-208. https://doi.org/10.19053/20275137.n18.2019.7385

Mastropierro Tellechea, María del Carmen. 2006. Archivos privados: Análisis y gestión. Buenos Aires: Alfagrama Ediciones.

Pené, Mónica Gabriela y Carolina Unzurrunzaga. 2015. "Archivos de escritores, bibliotecas universitarias y acceso abierto”, en IV Jornada abierta de la cátedra Desarrollo de la Colección y de los servicios de acceso. Buenos Aires: Julio Jatuf. http://www.juliodiazjatuf.com.ar/pdfs/Segunda_gacetilla_JADECSA_2015.pdf

Pollard, Riva A. 2001. "The Appraisal of Personal Papers: A Critical Literature Review”. Archivaria 52: 136-150. https://archivaria.ca/index.php/archivaria/article/view/12818/14029

Portela Filgueiras, Isabel. 2017. "El paradigma de los archivos personales: Estudio histórico-archivístico del fondo de Toribio del Campillo (1823-1900)”. Tesis de doctorado, Universidad Complutense de Madrid, Facultad de Filología. https://eprints.ucm.es/40737/

Prieto, Laura. 2016. "Valorización de archivos personales musicales mediante el sistema de catalogación relacional multidireccional: El ejemplo del Archivo Mantecón”. Documentación de las Ciencias de la Información 39: 241-282. https://doi.org/10.5209/DCIN.54418

Pulgarín Gallego, Marta Cecilia. 2017. "Revisión bibliográfica sobre archivos personales en Argentina, Brasil, Colombia, Cuba, España y México en el período 2006-2016". Trabajo de licenciatura, Universidad de Antioquia, Escuela Interamericana de Bibliotecología. http://hdl.handle.net/10495/9297 
Ramos Villalobos, Roxana Guadalupe. 2017. "Socorro Bastida y Muro: Una vida en la danza”. Entreciencias: Diálogos en la Sociedad del Conocimiento 5 (13): 63-76. https://doi.org/10.21933/J.EDSC.2017.13.200

RBM: A Journal of Rare Books, Manuscripts, and Cultural Heritage. 2004. The ARL "Exposing Hidden Collections" initiative 5 (2). https://rbm.acrl.org/index.php/rbm/issue/view/37

Ríos, Patricia. 2008. "La importancia de la organización y la conservación de un archivo personal”. Revista Universidad de Sonora (23): 52-55. http://www.revistauniversidad.uson.mx/revistas/23-11.pdf

Rosell León, Yorbelis. 2006. "Consideraciones teóricas sobre la transferencia de archivos personales e institucionales en Cuba: impacto de las tecnologías”. Acimed 14 (4). http://bvs.sld.cu/revistas/aci/vol14_4_06/aci12406.htm

Rucio Zamorano, María José. 2012. "Una puerta a la intimidad: Control técnico y acceso a los archivos personales de la Biblioteca Nacional de España”, en 5 as. Jornadas Archivando, coordinado por Javier González Cachafeiro, 9-24. León: Fundación Sierra Pambley.

https://dialnet.unirioja.es/servlet/articulo? codigo $=5117373$

Sanguinetti Epifanio, Néstor. 2018. "Los manuscritos de Delmira Agustini desde la crítica genética", en Actas de las II Jornadas de discusión y I Congreso internacional, "Los archivos personales: Prácticas archivísticas, problemas meteorológicos y usos historiográficos", compilado por María Virginia Castro y María Eugenia Sik, 324-339. Buenos Aires: CeDInCI.

http://jornadasarchivos.cedinci.org/wp-content/uploads/2019/02/Actas-Jornadas-Archivos-Personales-ilovepdf-compressed.pdf

Santos, Ana Rita Oliveira. 2018. "Aprendizagem em ação: contributos para a preservação do Arquivo Pessoal de Joaquim Falcão Marques Ferrer”. Trabajo de licenciatura, Universidade de Coimbra, Faculdade de Letras. http://hdl.handle.net/10316/82568

Santoyo Bastida, Beatriz. 2014. "Los archivos personales en México: su importancia, custodia y el valor de sus documentos como fuentes para la historia”. Bibliotecas y archivos 1 (3): 39-46.

https://biblat.unam.mx/es/revista/bibliotecas-y-archivos-mexico-d-f/articulo/ los-archivos-personales-en-mexico-su-importancia-custodia-y-el-valor-de-sus-documentos-como-fuentes-para-la-historia

Svicero, Thais Jeronimo. 2013. "Os arquivos pessoais e sua importância como patrimônio documental e cultural”. História e Cultura 2 (1): 221-237. https://doi.org/10.18223/hiscult.v2i1.869

Vautier, Marine. 2015. "Les archives personnelles, valeur, mémoire, émotions - Les archives d'une famille déracinée”. Tesis de maestría, Université Angers, Faculté des lettres, langues et sciences humaines. http://dune.univ-angers.fr/documents/dune5080

Yarrow, Alexandra, Barbara Clubb y Jennifer-Lynn Draper. 2009. Bibliotecas públicas, archivos y museos: tendencias de colaboración y cooperación. La Haya: IFLA. https://www.ifla.org/files/assets/hq/publications/professional-report/113.pdf

Zegers, Pablo. 2006. "El legado literario de Gabriela Mistral en el Archivo del Escritor de la Biblioteca Nacional de Chile", en Seminario de Archivos Personales, coordinado por Julián Martín Abad, 381-401. Madrid: Biblioteca Nacional. 
Para citar este texto:

Pené, Mónica Gabriela, Florencia Bossié, Victoria Calvente y Flavia Giménez. 2021. "Los archivos personales conservados en bibliotecas universitarias argentinas y su importancia para la investigación y la cultura”. Investigación Bibliotecológica: archivonomía, bibliotecología e información 35 (86): 143-160.

http://dx.doi.org/10.22201/iibi.24488321xe.2021.86.58272 